\title{
"What will happen when I can no longer cope?" Burden in caring for people with severe mental illness
}

\section{Zamzaliza Abdul Mulud}

School of Nursing and Midwifery, UCC

Mental illness is nothing to be ashamed of, but stigma and bias shame us all. (Bill Clinton)

\section{The case study}

Jane is a 55 year old woman, who cares for her son with schizophrenia. When Jane is asked about any difficulties in caring for her son, she becomes tearful as nobody has ever asked her about her feelings before. Jane believes that caring for her son is like carrying a heavy load because sometimes she cannot cope with the demands of caregiving and as a result she always feels overworked, stressed, angry, and dissatisfied. She also suffers a great deal of shame and embarrassment associated with her son's behaviour problems. Jane shared her experience of her son's behavioural problem :"Yesterday, he took off his clothes in the shopping mall, where there were a lot of people. It was such an embarrassing situation. Furthermore, the people around there were telling others that I am his mother. I felt so embarrassed". This case study reflects the common scenario experienced by caregivers of people with severe mental illness on the daily basis.

\section{Introduction}

Mental health problems affect millions of people worldwide. It affects not only patients, but their family members and the community as well. The de-institutionalisation of patients with mental illness from hospitals to community care has resulted in greater involvement of informal caregivers in the patient's management. Furthermore, due to the chronic characteristics of mental illnesses, patients often require long periods of care, thus caregivers are left with no choice other than adjusting their life to accommodate these changes. Some caregivers were able to adapt to the new role successfully, but others experienced a significant level of burden. As illustrated in the above case study, caring for individuals with mental illness proved to be more challenging than caring for individuals with other illness.

In the current health care system, informal caregivers of people with severe mental illness play a crucial role in influencing patients' prognosis and reducing rates of hospital admission, relapse and episodes of self-harm. However, studies of caregivers have shown that 
caring for individuals with severe mental illness often impacts all domains of caregivers' lives, including work, leisure, income, physical and emotional health and significant relationships with other people. Previous research findings have also shown that tiredness, sadness, constant stress, uncertainty and frustration were the common feelings of burden expressed by caregivers. In addition, unlike other chronic illnesses, the stigma often attached to mental illness (as illustrated in Figure 1) was a significant factor in greater caregiver burden and caused caring for people with mental illness to be a more stressful experience.

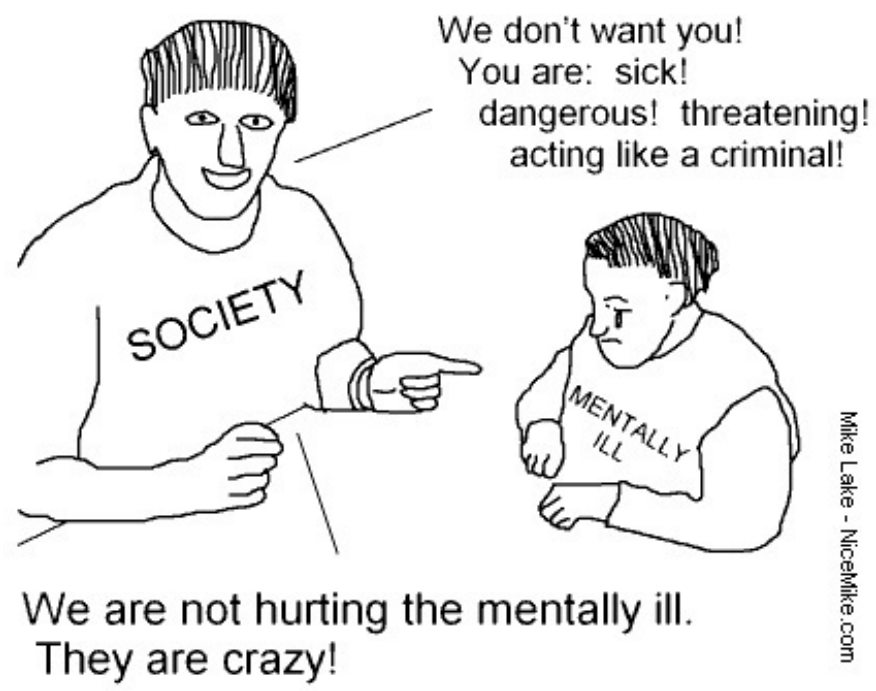

Figure 1: Stigma of mental illness — Source Mike Lake, www.nicemike.com

\section{Factors influencing caregiver burden}

It is widely accepted that the factors associated with caregiver burden are complex and multi-dimensional. Efforts are being made internationally to identify factors that contribute to caregiver burden so that the appropriate interventions, which are targeted towards these populations, can be identified. Caregiver burden has common causes (shown in Figure 2) and findings from previous studies suggested that caregivers who are older and female, those with depressive symptoms and with a higher self-perceived stigma were more likely to report a higher level of caregiver burden. Besides these, other factors that were found to be associated with caregiver burden include availability of social support. However, some of these factors are not conclusive in predicting caregiver burden. For example, a study in Malaysia among caregivers of patients with dementia found that low external support received by caregivers was associated with caregiver burden. Yet, contrary to the findings in the Malaysian study, a study done among Italian caregivers of patients with the same illness indicated that the lack of social support was seen as having not contributed to a burden. Thus, the cultural differences in caregiving and coping style may also reflect differences in the way caregiving affected caregivers from different ethnic groups and cultural backgrounds. 

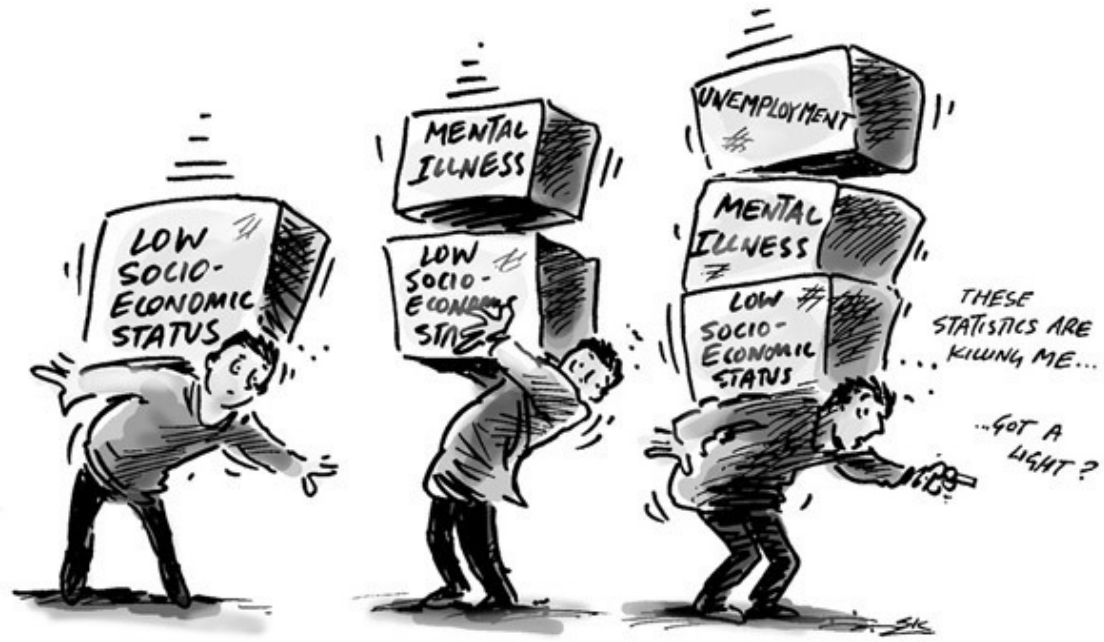

Figure 2: Multidimensional factors that contributed to burden in mental illness. Source: Simon Kneebone

Despite the inconsistencies in the literature about the factors that contributed to caregiver burden, resilience might be the key to explain how caregivers 'bounce back' and deal with challenges in caring for individuals with severe mental illness. Alongside caregiver burden, resilience is included in the present study in order to recognise the potential for personal growth associated with the caregiving role. This is another approach to understanding what caregivers experience in dealing with patients of mental illness, without denying the existence of burden and stress felt by caregivers. Previous studies among caregivers of elderly people with Alzheimer's disease, female caregivers of individuals with serious mental illness and child caregivers found that those who were more resilient reported low levels of burden in caregiving.

Nevertheless, in the Malaysian context, little is known about the experience of caregivers in caring for individuals with severe mental illness. There are few studies done on caregiving that focus on caregiver burden among patients with dementia and Parkinson disease, except for one qualitative study which was focused on caregiver burden among caregivers of patients with mental illness. The factors associated with caregiver burden and resilience are well established in Western countries and in some Asian countries. In Malaysia however, since the studies are limited in this area, little is known about the magnitude of caregiver burden and the impact of resilience among caregivers caring for individuals with severe mental illness. The paucity of research on caregiver burden and its associated factors among Malaysian caregivers of individuals with mental illness suggests that research is needed to address this issue. 


\section{My research}

\section{Aims and design}

The primary aim of this quantitative, correlational study design was to examine the association between caregiver burden and caregivers' demographic backgrounds, caregivingrelated stressors and resilience among the population being studied. The development of a conceptual framework for this study has been guided by the Pearlin Stress Process Model, and the Model of Carer Stress and Burden, as well as findings from a comprehensive literature review. In this conceptual framework, there are three factors that contribute to the outcome of caregiver burden, namely the background and context of caregiving, primary stressors and resilience. The sampling frame of this study was composed of a list of caregivers of individuals with severe mental illness attending Psychiatric Outpatient Clinics in Malaysia. Two clinics were selected as they serve as reference centres for severe mental illness for cases around West Malaysia. Self-administered questionnaires were used to collect data and at the end of the data collection period, 201 caregivers participated. The ethical approval to conduct this research was granted by the Medical Research and Ethics Committee, Malaysia and the University College Cork Clinical Research Ethics Committee.

\section{Findings}

Findings from the present research contribute to the growing evidence of the role of caregivers' socio-demographic variables, caregiving-related stressors and resilience towards caregiver burden. The results of my study indicate that caregiver burden was greater for older and female caregivers, members of the Chinese ethnic group who were unemployed, divorced or widowed, those with a perceived moderate health status and having a medical problem. On the other hand, caregiving-related stressors that were significantly associated with caregiver burden included longer time spent on caregiving activities, insufficient emotional support and support with caregiving tasks and assistance as well as patient behavioural disturbances. Finally, the findings also supported the hypothesis of the study as there was a significant relationship between resilience and caregiver burden. A moderate negative correlation indicates that increases in resilience correlated with decreases in the level of caregiver burden.

One interesting finding of this research highlighted that women caring for individuals with severe mental illness (like Jane, in the case study) were at higher risk of burden compared with male caregivers and this finding was supported by results from previous studies. The Role Theory suggests that an individual's social behaviour largely depends on society's expectation of the role that the person is assuming. Therefore, women who have always been considered as the caretaker must take up the role as a caregiver, whether they are willing or prepared to do so or not. Besides, female caregivers were more often subjected to stigma, guilt, caregiver strain and client dependency than their male counterparts- 
possibly explaining why female caregivers reported higher levels of burden.

Finally, it can be concluded that the caregiving burden is a common phenomenon that is shared by caregivers from various cultural backgrounds, although the contributing factors might differ from one population to another. The findings from this research also highlight the fact that mental illness affects not only patients but their family members as well. Thus, it is important to identify burden among caregivers caring for individuals with mental illness. Nurses or other health care providers need to be cognisant of caregivers' levels of burden and distress in caring for patients with severe mental illness. This evaluation should be done as part of an assessment, particularly when caregivers accompany patients for their follow up -or during the home visit. Furthermore, informal caregivers should be actively included in the patients' management and interventions so that the caregivers are able to recognise any changes in patient's behaviour and respond accordingly.

I would like to thank my supervisors Professor Geraldine McCarthy and Dr Mary Rose Day for their support and guidance. I wish to acknowledge the Ministry of Education, Malaysia and Universiti Teknologi MARA, Malaysia for the financial support of the study. 